\title{
Experimental System for Remote Airport Traffic Control Center Research
}

\author{
N. Fürstenau, M. Schmidt, M. Rudolph, M. Friedrich, C. Möhlenbrink, A. Papenfuß, \\ S. Kaltenhäuser
}

\author{
German Aerospace Center (DLR) \\ Institute of Flight Guidance \\ Braunschweig, Germany
}

\begin{abstract}
:
An experimental environment is described for research in remote surveillance and control of several small airports from a central location. Since 2004 a corresponding Remote Tower Operation (RTO) testbed has been realized by the German Aerospace Center at the Braunschweig research airport [1]. Technical as well as human factors aspects of the Remote Airport Traffic Control Center (RTC) are investigated within this environment [2][3]. Because small airports usually lack any electronic surveillance such as ground movement radar the basic philosophy consists in replacement of the controllers direct view out of the tower windows (far view) by a high resolution videopanorama system. This initial design is based on work and task analyses as well as results reported in the literature [4] which indicate the importance of the visual information for present days controller's work procedures.

The research environment consists of an experimental part for field testing technical components, verifying specifications and validating operational procedures under live as well as video replay conditions and simulation systems for repeatable experiments with the new work system under controlled conditions, allowing for quantitative evaluation of different technical and operational concepts.

The present $180^{\circ}$-video panorama system for live field tests consists of four digital high resolution CCD cameras located near Braunschweig tower, and a remotely controlled pan-tilt zoom (PTZ) camera (including automatic tracking option). The cameras are connected to PC clusters for compression, image processing / movement detection, decompression and panorama reconstruction with $4+1$ standard high resolution displays. A $450 \mathrm{~m}$ fiberoptic Gbit Ethernet link connects sensor and display clusters. Cameras are calibrated for geographically correct superimposition of relevant data for augmenting the (reconstructed) view out of the tower windows with flight data. Field testing of the reconstructed far view with participation of local controllers shows an effective visual resolution of 2 arcmin in agreement with the theoretical predictions. The PTZ camera provides a "foveal" vision component including object tracking options, with a high resolution exceeding the human eye (1 arcmin) within an observation angle $<15^{\circ}$. The replay function allows for detailed reconstruction of specific events. Full day video data (typically 500 GB) are stored on hard drives with 4.5 Tbyte memory. Video-see-through augmentation of the reconstructed outside view, e.g. by means of data from electronic non-visual sources superimposed on the far view of the cameras improves the controllers situational awareness. The augmented vision function also supports a compact RTO-work environment due to reduction of numbers of displays. As an extension of the local field test system a second more distant international airport controlled by DFS (the German ANSP) will be equipped and connected to the experimental system, to provide the framework for long distance and passive mode testing.

Two different simulation systems have been realized for support of the design of the RTO/RTC work environment by repeatable experiments with expert and non-expert human operators. One system is based on a standard $200^{\circ}$-airport tower-simulator (ATS) which was extended by a RTO-console with self made 31"-backprojection displays. The traffic as generated by the ATS-simulation engine and controlled by pseudopilots is be transferred into
\end{abstract}


the RTO-console panoramasystem after transformation into video format. Therefore the same videopanorama software as with the live video system can be used. This enables the integration of augmented vision features and image processing (e.g. movement detection) in the same way as with the live system. The only difference between a simulated and a real airport and traffic scenario is that pseudopilots instead of real ones interactively control the (simulated) aircraft. Simultaneously the simulated airport ground traffic can be displayed on the large $200^{\circ}$ - ATS-visualisation system. With different simulation engines for ATS and RTO systems a simulation scenario with remote control of a small airport (RTO) from a tower cab of a large airport (ATS) is realizable.

For part task simulation experiments with significantly reduced organizational and infrastructure effort a Petri-net based computer simulation "FairControl" [5] using a simplified and schematic airport microworld was realized. The Colored Petrinet as a kind of flexible state machine allows for dynamical discrete state traffic simulation which drives a graphical display and tesat subject's input/output menues. The traffic-process Petri net is coupled with a user interface (HMI) net. Optionally a decision model (controller agent) realizing a limited number of rule based decisions may be connected to the executable HMI model, so that the whole system can run autonomously. One unique possibility of such a discrete state system is a formal state space analysis and the comparison of user model behavior (decision making) with real operators.

Both simulation systems are usually operated together with one or two eye tracking analyzers (SMI-eyelink) with optical head tracking system, in order to monitor the operator's attention dynamics during decision making and task execution. In this way airport traffic situation and operator's behavior may be correlated for quantitative performance analysis of users in different RTO/RTC work environments.

[1] M. Schmidt, M. Rudolph, B. Werther, N. Fürstenau, Development of an Augmented Vision Videopanorama Human-Machine Interface for Remote Airport Tower Operation, Proc. HCII2007 Beijing, Lecture Notes Computer Science vol. 4558,Springer-Verlag Berlin (2007) pp. 1119-112

[2] N. Fürstenau, M. Schmidt, M. Rudolph, C. Möhlenbrink, W. Halle, "Augmented vision videopanorama system for remote airport tower operation", in: Proc. ICAS 2008, 26th Int. Congress of the Aeronautical Sciences. I. Grant (Ed.), Anchorage, (2008) ISBN 0-9533991-9-2

[3] M. Schmidt, M. Rudolph, A. Papenfuß, M. Friedrich, C. Möhlenbrink, S. Kaltenhäuser, N. Fürstenau. Remote airport traffic control center with augmented vision video panorama. 28th Digital Avionics Systems Conference.2009. DASC'09. IEEE/AIAA.23-29 Oct. 2009. ISBN 978-1-4244-4078-8. pp. 4.E.2-1 - 4.E.215.

[4] Tavanti, M. (2006). "Control Tower Operations: A literature review of task analysis studies". Eurocontrol Experimental Center, EEC Report, 2006/06

[5] Werther, B., Möhlenbrink, C., Rudolph, M., "Colored petri net based formal airport control model for simulation and analysis of airport control processes", In: V.G. Duffy (Ed.) Digital Human Modeling, Lect. Notes Computer Science vol.4561, Springer-Verlag Berlin Heidelberg (2007), pp. 1027-1036 